\title{
Aberrant Temporal-Spatial Complexity of Intrinsic Fluctuations in Major Depression
}

\section{Kaizhong Zheng}

Xi'an Jiaotong University https://orcid.org/0000-0002-9723-3227

\section{Baojuan Li}

Fourth Military Medical University

\section{Hongbing Lu}

Fourth Military Medical University

\section{Huaning Wang}

Fourth Military Medical University

\section{Baoyu Yan}

Fourth Military Medical University

\section{Karl Friston}

University of London

\section{Yuxia Wu}

Xi'an Jiaotong University

Jian Liu

Fourth Military Medical University

\section{Xi Zhang}

Fourth Military Medical University

\section{Mengwan Liu}

Fourth Military Medical University

\section{Liang Li}

Academy of Military Medical Sciences

\section{Badong Chen ( $\nabla$ chenbd@xjtu.edu.cn )}

Xi'an Jiaotong University

\section{Dewen Hu}

National University of Defense Technology

\section{Lingjiang Li}

Central South University

\section{Research Article}


Keywords: major depressive disorder (MDD), resting-state functional networks, co-activation pattern analysis, brain network dynamic, temporal-spatial complexity of intrinsic fluctuations

Posted Date: July 16th, 2021

DOl: https://doi.org/10.21203/rs.3.rs-698478/v1

License: (c) (1) This work is licensed under a Creative Commons Attribution 4.0 International License. Read Full License

Version of Record: A version of this preprint was published at European Archives of Psychiatry and Clinical Neuroscience on April 13th, 2022. See the published version at https://doi.org/10.1007/s00406022-01403-x. 


\section{Aberrant temporal-spatial complexity of intrinsic fluctuations in major}

\section{depression}

Kaizhong Zheng ${ }^{1, \#}$, Baojuan $\mathrm{Li}^{2, \#}$, Hongbing $\mathrm{Lu}^{2, \#}$ Huaning Wang ${ }^{3}$, Baoyu Yan ${ }^{2}$, Karl J.

Friston ${ }^{4}$, Yuxia $\mathrm{Wu}^{5}$, Jian $\mathrm{Liu}^{6}$, Xi Zhang ${ }^{2}$, Mengwan $\mathrm{Liu}^{2}$, Liang $\mathrm{Li}^{7}$, Badong Chen ${ }^{1, *}$, Dewen $\mathrm{Hu}^{8, *}$, Lingjiang $\mathrm{Li}^{9, *}$

${ }^{1}$ Institute of Artificial Intelligence and Robotics, Xi'an Jiaotong University, Xi'an, Shaanxi, 710032, China.

${ }^{2}$ School of Biomedical Engineering, Fourth Military Medical University, Xi'an, Shaanxi, 710032, China.

${ }^{3}$ Xijing Hospital, Fourth Military Medical University, Xi'an, Shaanxi, 710032, China.

${ }^{4}$ The Wellcome Department of Imaging Neuroscience, Institute of Neurology, University College London, 12 Queen Square, London WC1N 3AR, UK

${ }^{5}$ Department of Information and Communication Engineering, Xi'an Jiaotong University, 710032, China

${ }^{6}$ Network Center, Fourth Military Medical University, Xi'an, Shaanxi, 710032, China.

${ }^{7}$ Institute of Military Cognition and Brain Sciences, Academy of Military Medical Sciences, 27 Tai-Ping Road, 100850, Beijing, China

${ }^{8}$ Department of Intelligence Science and Technology, College of Intelligence Science and Technology, National University of Defense Technology, 410073, Changsha, China

${ }^{9}$ Department of Psychiatry, The Second Xiangya Hospital, Central South University, China 


\section{Address for Correspondence:}

Badong Chen ${ }^{1}$, Dewen $\mathrm{Hu}^{8}$, Lingjiang $\mathrm{Li}^{9}$

${ }^{1}$ Institute of Artificial Intelligence and Robotics, Xi'an Jiaotong University, Xi'an, Shaanxi, 710032, China.

${ }^{8}$ Department of Intelligence Science and Technology, College of Intelligence Science and Technology, National University of Defense Technology, Changsha, China.

${ }^{9}$ Department of Psychiatry, The Second Xiangya Hospital, Central South University, China Emails: chenbd@xjtu.edu.cn; dwhu@ nudt.edu.cn; LLJ2920@csu.edu.cn

\# These authors contributed equally. 


\section{Abstract}

Accumulating evidence suggested that the brain is highly dynamic, thus investigation of brain dynamics especially in brain connectivity would provide crucial information that stationary functional connectivity could miss. This study investigated temporal expressions of spatial modes within the default mode network (DMN), salience network $(\mathrm{SN})$ and cognitive control network $(\mathrm{CCN})$ using a reliable data-driven co-activation pattern (CAP) analysis. We found reduced number of CAPs, as well as transitions between different CAPs of the DMN and $\mathrm{CCN}$, in patients with MDD. These results suggested reduced variability and flexibility of these two brain networks in the patients. By contrast, we also found increased number of CAPs of the SN in the patients, indicating enhanced variability of the $\mathrm{SN}$ in individuals with MDD. In addition, the patients were characterized by prominent activation of mPFC and insula. More importantly, we showed that our findings were robust and reproducible with another independent data set. Our findings suggest that functional connectivity in the patients may not be simply attenuated or potentiated, but just alternating faster or slower among more complex patterns. The aberrant temporal-spatial complexity of intrinsic fluctuations reflects functional diaschisis of resting-state networks as characteristic of patients with MDD.

Keywords: major depressive disorder (MDD); resting-state functional networks; 
co-activation pattern analysis; brain network dynamic; temporal-spatial complexity of intrinsic fluctuations

\section{Introduction}

Major depressive disorder (MDD) is a prevalent psychiatric disorder characterized by the symptoms of depressed mood, anhedonia, cognitive impairments and disturbed sleep or appetite [1-4]. Researchers have implicated aberrant resting-state functional intrinsic networks in the pathophysiology of $\operatorname{MDD}[5,6]$. Specifically, functional neuroimaging studies have highlighted the involvement of abnormal functional connectivity within the default mode network (DMN), cognitive control network $(\mathrm{CCN})$ and salience network $(\mathrm{SN})$, etc [7-10] in MDD. However, findings from previous studies have been mixed. For example, the majority of studies have reported elevated DMN connectivity in the patients [11, 12], while some studies also observed decreased DMN connectivity in MDD [13, 14]. These inconsistent findings undermine the potential usefulness of resting state fMRI as an endophenotype or biomarker for major depressive disorder.

One of the possible contributing factors to the contradictory results may be the non-stationary characteristic of brain connectivity [15-18]. Accumulating evidence have shown that the patterns of brain connectivity can change within time scales of seconds to minutes $[16,19,20]$. This kind of nonstationary phenomenon has been 
consistently reported in unconscious anesthetized macaques, healthy controls, as well as patients with Parkinson's disease [21], autism [22], and depression [23]. Thus traditional (stationary) functional connectivity analysis which simply reports the averaged functional connectivity across the scanning session overlooks dynamics in brain connectivity, and hampers the capacity to capture the depression-specific alterations in brain dynamics.

Some attempts have been made to investigate temporal characteristics of functional connectivity among fixed predefined regions with the sliding-window algorithm which studies the temporal changes of functional connectivity in a truncated sequence of entire time series $[20,24,25]$. Altered variability of functional connectivity within the DMN and the frontoparietal network was observed in adults with depression [26]. Moreover, compared to healthy controls, patients with MDD exhibited similar functional connectivity states, but certain states were expressed for markedly different durations [27].

In addition to functional connectivity among fixed pre-defined regions, the spatial pattern that defines the intrinsic network is also highly dynamic. Recently, Liu et al. (2013) proposed the co-activation pattern (CAP) analysis, which provided a richer characterization of functional connectivity that may increase the sensitivity and specificity of dynamic functional network analysis [28, 29]. Most importantly, recent studies have shown that CAP analysis can establish the relationship between spatial 
patterns and temporal changes within intrinsic brain networks, unlike the approach of the sliding-window [28, 30-32]. However, it remains unclear whether temporal expression of spatial modes captured by the CAP analysis would be altered in patients with MDD.

In the current study, we aimed to investigate the temporal changes in spatial patterns within resting-state functional networks in 158 patients with MDD. We proposed a revised CAP analysis algorithm (spCAP). Compared to the original algorithm in Liu et al. (2013), the spCAP is able to automatically determine the optimal number of the CAP maps instead of arbitrary setting it to 8 [28]. In addition, the spCAP employed a spectral clustering algorithm which could accommodate skewed distribution of the sample distance. This algorithm was then used to extract CAPs of the DMN, SN and CCN in patients with MDD. The features including numbers of CAP maps, occurrence rate, within-cluster similarity, persistence and transitions of CAP maps were then compared between the MDD group and healthy controls. More importantly, these findings were reproducible and robust in another independent dataset. 


\section{Methods and Materials}

\subsection{Participants}

\section{Principal Data Set}

A total of 158 patients with major depressive disorder and 102 healthy controls participated in this study. All subjects were recruited from the Zhumadian Psychiatric Hospital. Patients with depression was clinically diagnosed using structural clinical interview for Diagnostic and Statistical Manual of Mental Disorders-IV (DSM-4). The inclusion criteria for MDD groups were as follow: 1) a Hamilton Depression scale (HAMD, 24 items) score of more than 20;2) no psychotropic medication for at least 2 weeks ( 6 weeks for fluoxetine) before inclusion. Exclusion criteria for both groups included current or history of systemic medical condition; brain injury; meeting the criteria of DSM-V alcohol/drug dependence in the past year, or meeting the criteria of DSM-V alcohol/substance abuse in the past 6 months; pregnant or breast breeding women; having used anticoagulants (heparin, warfarin, etc.), glucocorticoids or medications for thyroid diseases in the past 3 months; abnormal urine toxicology or thyroid screening results and significant current suicidal ideation or suicidal attempt. Table 1 show the demographical and clinical characteristics of the principal data set.

\section{Replication Data Set}

A total of 99 subjects were recruited including 43 MDD patients and 56 healthy 
controls. All the subjects were informed about the study's aims and procedures - and signed informed consent. The experiment was conducted in accordance with the requirements of the Ethics Committee in Xi-jing Hospital. Patients with MDD were recruited from the Department of Psychiatry in Xi-jing Hospital. All 43 MDD patients conformed to the diagnostic criteria of DSM-IV for a current episode of MDD - as assessed by two experienced psychiatrists. The inclusion criteria were as follow: a Hamilton Depression scale (HAMD, 24 items) score of more than 18; a of Hamilton anxiety scale (HAMA) score of more than 12. Exclusion criteria included: brain lacuna infarction, demyelinating disorder, mild cerebral atrophy, previous history of encephalitis, past mental abnormality, vascular malformation, incomplete HAMD test, left handedness, imaging contraindications due to artificial teeth, deficient cerebellum in fMRI coverage, shadow in T1 image, or problems in spatial normalization, abnormal T1 segmentation and inappropriate head position. Table 1 show the demographical and clinical characteristics of the replication data set.

\subsection{Data acquisition and preprocessing}

\section{Principal Data Set}

Resting-state fMRI data and T1-weighted data were collected in Zhumadian Psychiatric Hospital using a GE Healthcare 3.0T MR scanner (Signa HDxt scanning, Milwaukee, WI). The subjects were asked to close their eyes, stay awake and lie still in the scanner without performing any explicit task during the fMRI scanning session. 
The head axis was aligned with the middle axle of MRI machine and two sponges were used to fix the subjects' head - in order to suppress head movement during the experiment. 180 functional images were collected for each subject with the following parameters: $\mathrm{TR}=2000 \mathrm{~ms}, \mathrm{TE}=30 \mathrm{~ms}$, flip angle $=90^{\circ}, \mathrm{FOV}=220 \times 220 \mathrm{~mm}$, slice thickness $=4 \mathrm{~mm}$, spacing $=0.6 \mathrm{~mm}$, matrix size $=64 \times 64$, number of slices $=33$. The high-resolution 3D brain anatomical images using a T1-weighted BRAVO sequence were also collected with the following parameters: $\mathrm{TR}=6.8 \mathrm{~ms}$, $\mathrm{TE}=2.5 \mathrm{~ms}$, flip angle $=90^{\circ}$, slice thickness $=1 \mathrm{~mm}$, spacing $=0.0 \mathrm{~mm}$, matrix size $=256 \times 256$, FOV $=$ $256 \times 256 \mathrm{~mm}$, number of slices $=192$, turnover time $(\mathrm{TI})=1100 \mathrm{~ms}$.

The first ten volumes of the functional fMRI data were discarded for magnetic saturation. Then, realignment was performed to correct for head motion between fMRI images at different time point by translation and rotation. The high-resolution structural image was then co-registered with functional images and segmented into gray matter, white matter and cerebrospinal fluid (CSF). The deformation parameters from the structural image to the MNI (Montreal Neurological Institute) template were then used to normalize the resting-state fMRI images into a standard space. Additionally, a Gaussian filter with a half maximum width of $8 \mathrm{~mm}$ was used to smooth the functional images. Next, each participant's time series were band-pass filtered in the range of $0.01-0.08 \mathrm{~Hz}$ and we regressed out the effects of head motion, white matter and cerebrospinal fluid signals. Finally, the signal of each voxel was 
demeaned and then normalized by its standard deviation using the software of MATLAB (https://www.mathworks.com/).

\section{Replication Data Set}

Resting-state fMRI data were collected in Xi-jing Hospital using a GE Discovery MR750 3.0 T MRI system. 210 resting-state fMRI images were collected for each subject with the following parameters: repetition time (TR) $=2000 \mathrm{~ms}$, echo time (TE) $=30 \mathrm{~ms}$, flip angle $=90^{\circ}$, field of view $(\mathrm{FOV})=240 \times 240 \mathrm{~mm}$, matrix size $=64 \times 64$, number of slices $=45$, slice thickness $=3.5 \mathrm{~mm}$, spacing $=0.0 \mathrm{~mm}$. A high-resolution structural image was also collected with the following parameters: $\mathrm{TR}=8.2 \mathrm{~ms}, \mathrm{TE}=$ $3.2 \mathrm{~ms}, \mathrm{FOV}=256 \times 256 \mathrm{~mm}$, matrix $=256 \times 256$, flip angle $=12^{\circ}$, slice thickness $=1$ $\mathrm{mm}$, spacing $=0.0 \mathrm{~mm}$.

The first ten volumes of the functional fMRI data were discarded for magnetic saturation. The remaining functional fMRI data had been preprocessed for slice timing, correcting for head movement, normalization, smoothing $(6 \mathrm{~mm})$, temporally filtering $(0.01-0.08 \mathrm{~Hz})$ and head motion, white matter and CSF regression. Finally, the signal of each voxel was demeaned and then normalized by its standard deviation using the software of MATLAB (https://www.mathworks.com/). 


\subsection{Group independent component analysis (GICA)}

We used group independent component analysis of fMRI Toolbox (GIFT, http://mialab.mrn.org/software/gift/index.html, v3.0b) to decompose the fMRI images into spatially independent components. The number of independent components was first estimated using the minimum description length (MDL) criterion and we then decomposed the fMRI images into the estimated number of spatially independent components. Finally, the SN, DMN, left and right $\mathrm{CCN}$ were identified according to the resting-state network templates created by Smith [33].

\subsection{Spontaneous co-activation pattern analysis}

\section{The workflow of spontaneous co-activation pattern analysis}

Spontaneous activity in resting-state networks - including DMN, SN and bilateral $\mathrm{CCN}$ - were investigated using the co-activation pattern (CAP) analysis [28]. Figure 1 shows the workflow of the spontaneous co-activation pattern analysis. In this study, the spontaneous co-activation pattern analysis is mainly divided into five steps: first, we extracted time courses of region of interest (ROI) (Figure 1A). Second, we computed the optimal threshold to select the supra-threshold frames (Figure 1B). Third, the spectral clustering algorithm was applied to cluster the supra-threshold frames (Figure 1D). Fourth, we determined the optimal number of the clusters to obtain CAP maps. Fifth, we compared the temporal properties of CAP maps in both 
groups. Finally, we further extracted the overall dominant CAP maps (dCAP) within resting-state networks and compared the spatial expressions of dCAP in both groups (Figure 1E).

\section{Extraction of time courses of ROI}

For each network, we specified a $6 \mathrm{~mm}$-sphere ROI centered on the peak seed locations for the DMN, SN, LCCN and RCCN according to GICA analysis. Time courses from these 4 ROIs were extracted (Figure 1A).

\section{Computing the optimal threshold}

According to a previous study [28], we computed the optimal threshold. Firstly, the spatial correlation (Pearson's correlation coefficient) between the average of the first $\mathrm{n} \%$ (threshold) frames of each ROI and corresponding independent components using GICA was computed, resulting in a distribution of the spatial correlation $M$ according to the different threshold. Then we computed the gradient vector $G$ of $M$ :

$$
G(i)=\left\{\begin{array}{cc}
M(2)-M(1) & i=1 \\
\frac{M(i+1)-M(i-1)}{2} & 1<i<n \\
M(n)-M(n-1) & i=n
\end{array}\right.
$$

Finally, we computed the optimal threshold $\mathrm{n} \%$ based on the variation of $G$. According to the above procedures, we obtained the optimal thresholds of DMN, SN, LCCN and RCCN which included $12.9 \%, 7.6 \%, 11.2 \%$ and $10 \%$ for the principal data set (Figure 3-6A). In the replication data set, the optimal thresholds of DMN, SN, 
LCCN and RCCN including $8 \%, 5 \%, 7 \%$ and 5\% were selected (Supplementary Figure 1-4A).

\section{The spectral clustering algorithm}

Figure 1C showed data distribution of patients with MDD in the principal data set using the principal component analysis (PCA). Importantly, we observed that the distance among frames in the principal data set may exhibit skewed distribution which is consistent with previous study [34]. Since the spectral clustering method is independent on the explicit estimation of data distribution [35], we selected the spectral clustering to cluster and pool the supra-threshold frames. The steps of spectral clustering are as follows:

Firstly, a cross-correlation matrix $J$ was obtained by calculating the Pearson correlation coefficient between each pair of fMRI frames. A distance matrix $S$ denoted as $1-J$ was then used to represent the distances among the supra-threshold frames.

Next, adjacency matrix $W$ was constructed based on $S$ :

$$
W(i, j)=\exp \left(\frac{-S(i, j)^{2}}{2 \sigma^{2}}\right)
$$

where $\sigma$ denotes the width parameter of the gaussian kernel function and is set to 0.1 . We then defined the degree matrix $D$ which is a diagonal matrix:

$$
D(i, j)=\sum_{j=1}^{n} W(i, j)
$$


and constructed the Laplacian matrix $L=D^{-1 / 2} W D^{1 / 2}$. The eigenvalues of $L$ were calculated, and the eigenvalues were then sorted in descending order. We obtained the first $k$ (the number of clusters) eigenvalues and calculated corresponding eigenvectors $U=\left[\begin{array}{lll}u_{1}, u_{2} \ldots & u_{k}\end{array}\right]$. Finally, the K-means algorithm was applied to cluster these frames according to the $U$ matrix.

\section{The determination of the optimal number of the clusters}

Moreover, we adopted the Calinski Harabasz' $(\mathrm{CH})$ index to identify the optimal number of the clusters [36]. $\mathrm{CH}$ is defined as:

$$
C H=\frac{S S_{B}}{k-1} / \frac{S S_{W}}{n-k}
$$

where $n$ denotes the total number of frames, $k$ denotes the number of clusters, $\mathrm{SS}_{\mathrm{W}}$ denotes the overall within-cluster variance and $\mathrm{SS}_{\mathrm{B}}$ denotes the overall between-cluster variance.

Based on a previous study [28], the optimal number of the clusters is not more than 20 . Thus, the spectral clustering approach was repeated with $\mathrm{k}=2, \ldots, 20$, and the optimal number of the clusters was selected on the basis of the highest Calinski Harabasz' values. Finally, the CAP maps were generated through averaging the frames assigned to the same cluster (Figure 1D).

\section{The temporal properties of CAP maps}

In addition, we compared the temporal properties of CAP maps including the 
occurrence rate of distinct CAPs, within-cluster similarity of CAP maps in both groups. The occurrence rate is defined as the proportion of the number of different CAP maps to the total number of frames in a subject and within cluster similarity is defined as the average of the Pearson correlation coefficient between each fMRI frames and corresponding CAP in a subject. We also compared the transitions of CAP maps: total frequency of CAP-to-CAP transitions from one frame to the next.

\section{The spatial expression of the overall dominant CAP maps}

To select the most reproducible pattern of CAP maps, the overall dominant CAP maps (dCAP) within resting-state networks were extracted (Figure 1E). Firstly, we computed the temporal fractions (TF) and distinct CAP maps were then sorted in descending order according to TF. The TF is defined as the proportion of the number of fMRI frames assigned to the same cluster to the total number of fMRI frames. Next, the series of temporal frames averages $S_{m}$ was computed:

$$
S_{m}=\sum_{1 \leq i \leq m} S M_{i} \times T F_{i}
$$

where $S M_{i}$ is the spatial map of $C A P^{i}$. Then we calculated the Pearson correlation coefficient $r_{s} i$ between $S M_{i}$ and the overall average of all frames. Finally, to remove miscellaneous CAPs, we selected the overall dominant CAP maps $\left(S M_{i}\right)$ through comparing $r_{s} i$ greater than the fixed threshold, where the fixed threshold was chosen as 0.95 according to a previous study [30]. 


\subsection{Statistical test}

Permutation test was used to assess the statistical significance of the number of CAP maps within three networks in both groups. In permutation testing, the class labels were randomly shuffled across participants 1,000 times (the principal data set and the replication data set) when guaranteeing the number of participants in each cohort invariant. Each time, the spontaneous co-activation pattern analysis was repeated, gaining the changes of number of CAP maps within three networks in both groups. The $p$ value was then defined as the proportion of the changes of number of CAP maps that were greater than the changes of number of CAP maps without permutation.

In addition, two sample $t$ test $(p<0.05)$ was applied to determine whether the occurrence rate of distinct CAP maps, within-cluster similarity and transitions of the CAP maps were significantly different between patients with MDD and healthy controls.

\section{Results}

\subsection{Spatial patterns of the resting-state networks and the time courses of regions of interest (ROIs)}

Thirty independent components were obtained by the GICA analyses. The DMN, bilateral CCN and SN were identified according to the spatial maps provided in Smith 
et al. 2009 [33]. The spatial patterns of these networks are shown in Supplementary Figure 1A. The coordinates of the peak seed locations (the left precuneus (MNI coordinates: $0,-60,15)$, the left middle cingulum (MNI coordinates: $0,24,36$ ), the left middle frontal gyrus $(-36,21,51)$, the right middle frontal gyrus $(39,21,51))$ are shown in Supplementary figure 1A. More details are listed in supplementary materials (Supplementary Table 1).

\subsection{Temporal expression of the CAP maps within three networks}

In this study, we examined the temporal properties of CAP maps within DMN, SN, LCCN and RCCN by computing the number of clusters, the occurrence rate of distinct CAP maps, transitions of CAP maps and within-cluster similarity of CAP maps. Figure 2-5 depict the spatial patterns and the temporal properties of CAP maps within DMN, SN, LCCN and RCCN in both groups.

Figure 2-5 B demonstrated that MDD patients differed significantly from healthy controls in the number of associated clusters. By CAP analysis, we observed that the DMN pattern was decomposed into 2 CAP maps in patients with MDD, while that of healthy controls was decomposed into 4 CAP maps (Figure 2B). Permutation test was further applied to assess the statistical significance of the number of CAP maps within DMN. We found significantly attenuated number of CAP maps within DMN in patients with MDD compared with healthy controls (permutation testing using 1,000 permutations, $p<0.001)$. Furthermore, $\mathrm{SN}, \mathrm{LCCN}$ and $\mathrm{RCCN}$ were further analyzed: 
the SN pattern was decomposed into $9 \mathrm{SN}-\mathrm{CAP}$ maps in patients with MDD, while the SN pattern of healthy controls was decomposed into 2 CAP maps (permutation testing using 1,000 permutations, $p<0.001$, Figure 3B), the $\mathrm{LCCN}$ pattern was decomposed into 2 LCCN-CAP maps in patients with MDD and healthy controls (permutation testing using 1,000 permutations, $p=0.007$, Figure 4B) and the $\mathrm{RCCN}$ pattern was decomposed into 2 RCCN-CAP maps in patients with MDD, while the RCCN pattern of healthy controls was decomposed into 3 CAP maps (permutation testing using 1,000 permutations, $p<0.001$, Figure 5B). Enhanced number of clusters within SN and attenuated number of clusters within DMN and LCCN indicated abnormal dynamic activation of spatial organization.

\subsection{Spatial expression of the overall dominant CAP maps}

In addition, we further investigated spatial expression of the overall dominant CAP maps to select the most reproducible pattern of CAP maps. Specifically, the overall dominant CAP, which is the CAP of the maximum temporal fractions, is able to reflect the most repeated spatial pattern dominating the brain repertoire [30]. Figure 6 depicts the spatial expression of the overall dominant CAP maps within distinct resting-state networks. Apparently, their overall dominant CAP maps within resting-state networks are similar (Figure 6C). 


\subsection{Reproducible test}

In the replication data set, we also used the GICA analyses to obtain twenty-seven independent components. Similarly, the spatial patterns of these networks are shown in Supplementary Figure 1B. The coordinates of the peak seed locations (left cuneus (MNI coordinates: 0, -72, 33), left anterior cingulum (MNI coordinates: -3, 27, 27), left middle frontal gyrus (MNI coordinates: -39, 27, 39) and right middle frontal gyrus (MNI coordinates: 39, 27, 39)) for the three resting-state networks are also shown in the figure 1B. More details are available in supplementary materials (Supplementary Table 2).

To test the reproducibility of findings from the principal data set, we further repeated the spontaneous co-activation pattern analysis on the replication data set. Similar observations of the number of clusters were observed in the replication data set: the DMN pattern was decomposed into 5 DMN-CAP maps in patients with MDD, while the DMN pattern of healthy controls was decomposed into 14 CAP maps (permutation testing using 1,0000 permutations, $p<0.0001$, Supplementary Figure 2B); the SN pattern was decomposed into $15 \mathrm{SN}$-CAP maps in patients with MDD, while the SN pattern of healthy controls was decomposed into 3 CAP maps (permutation testing using 1,0000 permutations, $p<0.0001$, Supplementary Figure 3B); the LCCN pattern was decomposed into 2 LCCN-CAP maps in patients with MDD, while the LCCN pattern of healthy controls was decomposed into 9 CAP maps (permutation 
testing using 1,0000 permutations, $p=0.0019$, Supplementary Figure 4B) and the RCCN pattern was decomposed into 7 RCCN-CAP maps in patients with MDD, while the RCCN pattern of healthy controls was decomposed into 10 CAP maps (permutation testing using 1,0000 permutations, $p=0.0278$, Supplementary Figure 5B). These results further supported our main findings: MDDs are characterized by enhanced number of clusters within $\mathrm{SN}$ and attenuated number of clusters within DMN and RCCN.

In addition, similar findings were observed in the results of occurrence rate and within-cluster similarity, DMN-CAP was detected to occur more frequently in healthy controls compared to patients with MDD (two-sample $t$ test, $t=2.266, p=0.0257$, uncorrected, Supplementary Figure 2C) and SN-CAP was observed to occur more frequently in MDD subjects (two-sample $t$ test, $t=3.664, p=0.0004$, uncorrected, Supplementary Figure 3C). However, results that significantly reduced within-cluster similarity of the DMN was observed in patients with MDD in the replication data set (two-sample $t$ test, $t=2.857, p=0.0052$, uncorrected, Supplementary Figure 2C), were missing from the principal data set. Moreover, increased transitions of CAP maps (two-sample $t$ test, $t=2.959, p=0.0039$, uncorrected, Supplementary Figure 3B) within SN in patients with MDD were also observed in the replication data set.

We also analyzed the spatial expressions of the overall dominant CAP maps within different resting-state networks in both groups. Similar spatial expressions of 
the overall dominant CAP maps of three networks were detected in the replication data set (Figure 6).

\section{Discussion}

In this study, we investigated how temporal expressions of spatial modes were altered in patients with MDD through the CAPs analysis. We found reduced number of CAPs, of the DMN and CCN in subjects with MDD, which suggested reduced variability in dynamic configuration of these two networks. In addition, the number of transitions between different CAPs was also significantly decreased in the patients, indicating reduced flexibility of the $\mathrm{DMN}$ and $\mathrm{CCN}$ in the patients. However, the variability and flexibility of brain dynamics of another core brain network (the $\mathrm{SN}$ ) was increased in the patients, suggesting enhanced spatial complexity of intrinsic fluctuations. More importantly, with another independent data set, we showed that our results were robust and reproducible.

The number of CAPs reflects the spatial complexity of temporal changes in resting-state intrinsic functional networks. Our findings of reduced number of CAP maps within DMN and CCN in patients with MDD suggested reduced variability in dynamic configuration of these two networks. These findings are in line with previous studies using sliding-window approach that have reported reduced dynamic functional connectivity among PFC subregions [37], or between the CCN and DMN [38], or reduced variability in dynamic functional network connectivity between the DMN and 
right CEN (rCEN) in patients with MDD [23]. Using a data-driven approach, our study further confirmed that variability of the DMN and CCN was disrupted in patients with MDD as reflected by decreased number of recurring coactivation maps. More importantly, our reproducible test with another independent data set showed that reduced number of CAPs may serve as a robust neuroimaging biomarker in the patients.

Distinct CAP maps correspond to distinct spatial coactivation patterns which are reflective of synchronization of fMRI signals [28]. The CAPs mode of the DMN that recurred most frequently in patients with MDD was CAP2 which showed more prominent activation in $\mathrm{mPFC}$ than other DMN-CAPs. This CAP had the maximum temporal fractions of $83.98 \%$, reflecting the most repeated spatial pattern dominating the brain repertoire [30]. The $\mathrm{mPFC}$ is a core region of the anterior subnetwork of the DMN. This region has been shown to be associated with social cognition involving the monitoring of one's own psychological states, and mentalizing about the psychological states of others $[39,40]$. Thus more prominent $\mathrm{mPFC}$ activation and prolonged expression of CAP2 in MDD subjects may account for maladaptive rumination- the process of repetitively and passively thinking about one's negative feelings, possible causes, and consequences in the patients [41, 42].

In addition to reduced number of CAPs, we also observed fewer transitions between these CAP modes in the patients, suggesting reduced flexibility of the DMN 
in MDD subjects. Transitions between CAPs are reflective of transitions between different brain states. Thus, our results suggested that the brain of the patients tend to stay longer of recurred more frequently in a brain state with prominent $\mathrm{mPFC}$ activation, and less frequently switched to other brain states. Considering that the mPFC is generally associated self-focus, fewer switching between CAP2 and other CAPs may result in the risk of deficits in the patients' ability to distinguish between the internal world and external environment [43, 44].

In contrast to the $\mathrm{DMN}$ and $\mathrm{CCN}$, the $\mathrm{SN}$ of the patients demonstrated increased number of recurring patterns in the patients, suggesting enhanced spatial complexity of intrinsic fluctuations. This finding is in line with previous studies, in which altered distribution of community assignment and flexibility of the ACC (anterior cingulate cortex) within SN in patients with MDD [45]. Although the dominant pattern of the SN showed considerable similarity between healthy controls and the patients. This dominant pattern encompassed anterior cingulate cortex, the right frontal gyrus and the left frontal gyrus. However, we also noticed prominent activation of the insula in several CAPs in the patients group. A growing literature supports the notion that SN drives the switching between activation of DMN and of CEN [46, 47]. And the insula is believed to be provided with specific function that assists in switching between functional networks $[48,49]$. Our findings indicated that abnormal dynamic activation of insula may lead to difficulties in switching between DMN and CEN in patients with MDD. 


\section{Limitations}

There are some limitations in this work. In the current study, we were interested in three intrinsic brain networks that have consistently been reported to be involved in MDD. Our study demonstrated deficits in brain dynamics of the DMN, SN and CCN. In future studies, we would extend our analysis to other resting-state brain networks such as the attention network, the motor network, and the visual network, etc. Recent studies showed that the functions supported by these networks were also damped in patients with MDD as well [50-52]. However, it is not clear if whether brain coactivation patterns would also be impaired in the patients. In addition, the subject in the current study were adults between the ages of 18 and 61. Although the results are robust in another independent data set, whether our findings are also reproducible in adolescent with MDD or patients with late onset MDD remains unknown. Our future work will further test the consistency of our findings in different age groups.

\section{Conclusions}

In conclusion, these results suggest abnormally temporal and spatial changes of functional networks that standard (stationary) functional connectivity could miss; functional connectivity in the patients may not simply attenuated or potentiated, but just alternating faster or slower among more complex patterns. An abrupt switch of brain patterns may lead to significant considerable variations of standard functional connectivity, which may be a crucial reason for the contradictory results reported in 
previous studies. The aberrant spatial complexity of intrinsic fluctuations reflects functional diaschisis of resting-state networks as characteristic of patients with MDD.

\section{Declarations}

\section{Ethics approval and consent to participants}

This study was approved by the Ethics Committee in Zhumadian Psychiatric Hospital and $\mathrm{Xi}$-jing Hospital. All subjects gave informed consent before participating in this study.

\section{Consent for publication}

Last version of the manuscript was approved by the authors before the submission.

\section{Availability of data and code}

The data that support the findings of this study as well as code used during the current study are available from the corresponding author upon reasonable request.

\section{Competing Interests}

All authors declare that they have no competing interests.

\section{Funding information}

This work was supported by the National Natural Science Foundation of China 
(61976248, 91648208, 61976175, 81371478, 61503411).

\section{Author's contributions}

All authors contributed to the study conception and design. Data collection was performed by the prof. Lingjiang Li and Dr. Kaizhong Zheng. The first draft of the manuscript was written by Dr. Kaizhong Zheng and Prof. Baojuan Li. All authors commented on previous versions of the manuscript. Dr. Kaizhong Zheng, Prof. Badong Chen and Prof. Baojuan Li reviewed and revised the manuscript. All authors approved the final manuscript as submitted.

\section{Acknowledgments}

Not applicable.

\section{Compliance with Ethical Standards}

Not applicable.

\section{Research involving Human Participants and/or Animals}

Human and human samples were used in the current study.

\section{References}

1. Song Z, Zhang M, Huang P (2016) Aberrant emotion networks in early major depressive disorder patients: an eigenvector centrality mapping study. Transl Psychiatry 6:e819. https://doi.org/10.1038/tp.2016.81

2. Belmaker RH, Agam G (2008) Major depressive disorder. N Engl J Med 358:55-68 
3. Fava M, Kendler KS (2000) Major depressive disorder. Neuron 28:335-341. https://doi.org/10.1016/S0896-6273(00)00112-4

4. Otte C, Gold SM, Penninx BW, et al (2016) Major depressive disorder. Nat Rev Dis Prim 2:16065. https://doi.org/10.1038/nrdp.2016.65

5. Mulders PC, van Eijndhoven PF, Schene AH, et al (2015) Resting-state functional connectivity in major depressive disorder: A review. Neurosci Biobehav Rev 56:330-344. https://doi.org/10.1016/j.neubiorev.2015.07.014

6. Ye M, Yang T, Qing P, et al (2015) Changes of Functional Brain Networks in Major Depressive Disorder: A Graph Theoretical Analysis of Resting-State fMRI. PLoS One 10:e0133775. https://doi.org/10.1371/journal.pone.0133775

7. Liu C-H, Guo J, Lu S-L, et al (2018) Increased Salience Network Activity in Patients With Insomnia Complaints in Major Depressive Disorder. Front psychiatry 9:93. https://doi.org/10.3389/fpsyt.2018.00093

8. Dutta A, McKie S, Deakin JFW (2014) Resting state networks in major depressive disorder. Psychiatry Res 224:139-151. https://doi.org/10.1016/j.pscychresns.2014.10.003

9. Sheng J, Shen Y, Qin Y, et al (2018) Spatiotemporal, metabolic, and therapeutic characterization of altered functional connectivity in major depressive disorder. Hum Brain Mapp 39:1957-1971. https://doi.org/10.1002/hbm.23976

10. Wang Y, Yang S, Sun W, et al (2016) Altered functional interaction hub between affective network and cognitive control network in patients with major depressive disorder. Behav Brain Res 298:301-309. https://doi.org/10.1016/j.bbr.2015.10.040

11. Li B, Liu L, Friston KJ, et al (2013) A treatment-resistant default mode subnetwork in major depression. Biol Psychiatry 74:48-54. https://doi.org/10.1016/j.biopsych.2012.11.007

12. Ho TC, Connolly CG, Henje Blom E, et al (2015) Emotion-Dependent Functional Connectivity of the Default Mode Network in Adolescent Depression. Biol Psychiatry 78:635-646. https://doi.org/10.1016/j.biopsych.2014.09.002

13. Bluhm R, Williamson P, Lanius R, et al (2009) Resting state default-mode network connectivity in early depression using a seed region-of-interest analysis: decreased connectivity with caudate nucleus. Psychiatry Clin Neurosci 63:754-761. https://doi.org/10.1111/j.1440-1819.2009.02030.x

14. Yan CG, Chen X, Li L, et al (2019) Reduced default mode network functional connectivity in patients with recurrent major depressive disorder. Proc Natl Acad Sci 116:201900390

15. Allen EA, Damaraju E, Plis SM, et al (2014) Tracking whole-brain connectivity dynamics in the resting state. Cereb Cortex 24:663-676. https://doi.org/10.1093/cercor/bhs352

16. Chang C, Glover GH (2010) Time-frequency dynamics of resting-state brain connectivity 
measured with fMRI. Neuroimage 50:81-98. https://doi.org/10.1016/j.neuroimage.2009.12.011

17. Smith SM, Miller KL, Moeller S, et al (2012) Temporally-independent functional modes of spontaneous brain activity. Proc Natl Acad Sci U S A 109:3131-3136.

https://doi.org/10.1073/pnas.1121329109

18. Di X, Biswal BB (2020) Intersubject consistent dynamic connectivity during natural vision revealed by functional MRI. Neuroimage 216:116698.

https://doi.org/10.1016/j.neuroimage.2020.116698

19. Kang J, Wang L, Yan C, et al (2011) Characterizing dynamic functional connectivity in the resting brain using variable parameter regression and Kalman filtering approaches. Neuroimage 56:1222-1234. https://doi.org/10.1016/j.neuroimage.2011.03.033

20. Hutchison RM, Womelsdorf T, Gati JS, et al (2013) Resting-state networks show dynamic functional connectivity in awake humans and anesthetized macaques. Hum Brain Mapp 34:2154-2177. https://doi.org/10.1002/hbm.22058

21. Fiorenzato E, Strafella AP, Kim J, et al (2019) Dynamic functional connectivity changes associated with dementia in Parkinson's disease. Brain 142:2860-2872. https://doi.org/10.1093/brain/awz192

22. Watanabe T, Rees G (2017) Brain network dynamics in high-functioning individuals with autism. Nat Commun 8:16048. https://doi.org/10.1038/ncomms16048

23. Wang J, Wang Y, Huang H, et al (2020) Abnormal dynamic functional network connectivity in unmedicated bipolar and major depressive disorders based on the triple-network model. Psychol Med 50:465-474. https://doi.org/10.1017/S003329171900028X

24. Handwerker DA, Roopchansingh V, Gonzalez-Castillo J, Bandettini PA (2012) Periodic changes in fMRI connectivity. Neuroimage 63:1712-1719. https://doi.org/10.1016/j.neuroimage.2012.06.078

25. Jones DT, Vemuri P, Murphy MC, et al (2012) Non-stationarity in the 'resting brain's' modular architecture. PLoS One 7:e39731. https://doi.org/10.1371/journal.pone.0039731

26. Demirtaş M, Tornador C, Falcón C, et al (2016) Dynamic functional connectivity reveals altered variability in functional connectivity among patients with major depressive disorder. Hum Brain Mapp 37:2918-2930. https://doi.org/10.1002/hbm.23215

27. Zhi D, Calhoun VD, Lv L, et al (2018) Aberrant Dynamic Functional Network Connectivity and Graph Properties in Major Depressive Disorder. Front Psychiatry 9:1-11. https://doi.org/10.3389/fpsyt.2018.00339

28. Liu X, Duyn JH (2013) Time-varying functional network information extracted from brief instances of spontaneous brain activity. Proc Natl Acad Sci U S A 110:4392-4397. https://doi.org/10.1073/pnas.1216856110 
29. Marshall E, Nomi JS, Dirks B, et al (2020) Coactivation pattern analysis reveals altered salience network dynamics in children with autism spectrum disorder. Netw Neurosci 4:1219-1234. https://doi.org/10.1162/netn_a_00163

30. Chen JE, Chang C, Greicius MD, Glover GH (2015) Introducing co-activation pattern metrics to quantify spontaneous brain network dynamics. Neuroimage 111:476-488. https://doi.org/10.1016/j.neuroimage.2015.01.057

31. Xue W, Kang J, Bowman FD, et al (2014) Identifying functional co-activation patterns in neuroimaging studies via poisson graphical models. Biometrics 70:812-822. https://doi.org/10.1111/biom.12216

32. Messé A, Hütt M-T, Hilgetag CC (2018) Toward a theory of coactivation patterns in excitable neural networks. PLoS Comput Biol 14:e1006084. https://doi.org/10.1371/journal.pcbi.1006084

33. Smith SM, Fox PT, Miller KL, et al (2009) Correspondence of the brain's functional architecture during activation and rest. Proc Natl Acad Sci U S A 106:13040-13045. https://doi.org/10.1073/pnas.0905267106

34. Liu X, Chang C, Duyn JH (2013) Decomposition of spontaneous brain activity into distinct fMRI co-activation patterns. Front Syst Neurosci 7:101. https://doi.org/10.3389/fnsys.2013.00101

35. Xiang T, Gong S (2008) Spectral clustering with eigenvector selection. Pattern Recognit 41:1012-1029. https://doi.org/10.1016/j.patcog.2007.07.023

36. Lukasik S, Kowalski PA, Charytanowicz M, Kulczycki , Vancouver, 24-29 July 2016, pp. 2724-2728 PBT-2016 IC on EC (2016) Clustering using flower pollination algorithm and Calinski-Harabasz index

37. Cui G, Wang Y, Wang X, et al (2020) Static and dynamic functional connectivity of the prefrontal cortex during resting-state predicts self-serving bias in depression. Behav Brain Res 379:112335. https://doi.org/10.1016/j.bbr.2019.112335

38. Yao Z, Shi J, Zhang Z, et al (2019) Altered dynamic functional connectivity in weakly-connected state in major depressive disorder. Clin Neurophysiol 130:

39. Kaiser RH, Whitfield-Gabrieli S, Dillon DG, et al (2016) Dynamic Resting-State Functional Connectivity in Major Depression. Neuropsychopharmacol Off Publ Am Coll Neuropsychopharmacol 41:1822-1830. https://doi.org/10.1038/npp.2015.352

40. Broyd SJ, Demanuele C, Debener S, et al (2009) Default-mode brain dysfunction in mental disorders: a systematic review. Neurosci Biobehav Rev 33:279-296. https://doi.org/10.1016/j.neubiorev.2008.09.002

41. Zamoscik V, Huffziger S, Ebner-Priemer U, et al (2014) Increased involvement of the parahippocampal gyri in a sad mood predicts future depressive symptoms. Soc Cogn Affect 
Neurosci 9:2034-2040. https://doi.org/10.1093/scan/nsu006

42. Nolen-hoeksema S, Wisco BE, Lyubomirsky S (2008) Rethinking Rumination. 3:400-424

43. Nelson B, Whitford TJ, Lavoie S, Sass LA (2014) What are the neurocognitive correlates of basic self-disturbance in schizophrenia? Integrating phenomenology and neurocognition: Part 2 (aberrant salience). Schizophr Res 152:20-27. https://doi.org/10.1016/j.schres.2013.06.033

44. Carhart-Harris RL, Leech R, Erritzoe D, et al (2013) Functional connectivity measures after psilocybin inform a novel hypothesis of early psychosis. Schizophr Bull 39:1343-1351. https://doi.org/10.1093/schbul/sbs117

45. Zheng H, Li F, Bo Q, et al (2018) The dynamic characteristics of the anterior cingulate cortex in resting-state fMRI of patients with depression. J Affect Disord 227:391-397. https://doi.org/10.1016/j.jad.2017.11.026

46. Chen AC, Oathes DJ, Chang C, et al (2013) Causal interactions between fronto-parietal central executive and default-mode networks in humans. Proc Natl Acad Sci U S A 110:19944-19949. https://doi.org/10.1073/pnas.1311772110

47. Goulden N, Khusnulina A, Davis NJ, et al (2014) The salience network is responsible for switching between the default mode network and the central executive network: replication from DCM. Neuroimage 99:180-190. https://doi.org/10.1016/j.neuroimage.2014.05.052

48. Deen B, Pitskel NB, Pelphrey KA (2011) Three systems of insular functional connectivity identified with cluster analysis. Cereb Cortex 21:1498-1506.

https://doi.org/10.1093/cercor/bhq186

49. Chang LJ, Yarkoni T, Khaw MW, Sanfey AG (2013) Decoding the role of the insula in human cognition: functional parcellation and large-scale reverse inference. Cereb Cortex 23:739-749. https://doi.org/10.1093/cercor/bhs065

50. Ho TC, Wu J, Shin D, et al (2013) Altered Cerebral Perfusion in Executive, Affective, and Motor Networks During Adolescent Depression. J Am Acad Child Adolesc Psychiatry 52:1076-1091.e2

51. Chen H, Liu K, Zhang B, et al (2019) More optimal but less regulated dorsal and ventral visual networks in patients with major depressive disorder. J Psychiatr Res 110:172-178

52. Ding YD, Yang R, Yan CG, et al (2021) Disrupted hemispheric connectivity specialization in patients with major depressive disorder: Evidence from the REST-meta-MDD Project. J Affect Disord 284: 


\section{Tables and Figures Legends}

Table 1. The Demographic and Clinical Characteristics of the participants.

\begin{tabular}{|c|c|c|c|c|c|c|}
\hline & \multicolumn{3}{|c|}{ Principal Data Set } & \multicolumn{3}{|c|}{ Replication Data Set } \\
\hline & $\operatorname{MDD}(n=158)$ & $\mathrm{HC}(\mathrm{n}=102)$ & $P$ value & $\operatorname{MDD}(n=43)$ & $\mathrm{HC}(\mathrm{n}=56)$ & $P$ value \\
\hline Age (years) & $36.1(10.0)$ & $32.5(8.3)$ & $0.003^{\mathrm{a}}$ & $35.2(11.2)$ & $32.3(10.8)$ & $0.19^{\mathrm{a}}$ \\
\hline Age range(years) & $18-58$ & $18-50$ & - & $17-61$ & $20-61$ & - \\
\hline Gender & $(68 \mathrm{M} / 90 \mathrm{~F})$ & $(58 \mathrm{M} / 44 \mathrm{~F})$ & $0.0315^{\mathrm{b}}$ & $(13 \mathrm{M} / 30 \mathrm{~F})$ & $(30 \mathrm{M} / 26 \mathrm{~F})$ & $0.02^{\mathrm{b}}$ \\
\hline Education (years) & $10.1(3.3)$ & $11.7(3.5)$ & $<0.01^{\mathrm{a}}$ & $11.4(3.3)$ & $15.8(4.3)$ & $<0.01^{\mathrm{a}}$ \\
\hline Handedness & $(158 \mathrm{R} / 0 \mathrm{~L})$ & $(102 \mathrm{R} / 0 \mathrm{~L})$ & $0^{\mathrm{b}}$ & $(43 \mathrm{R} / 0 \mathrm{~L})$ & $(56 \mathrm{R} / 0 \mathrm{~L})$ & $0^{\mathrm{b}}$ \\
\hline
\end{tabular}

Notes: Demographic information for each variable is expressed as mean (standard deviation) or count. MDD:

Major depressive disorder; HC: Healthy controls; F, female; M, male; R, right handedness; L, left handedness.

${ }^{\mathrm{a}}$ represents $\mathrm{P}$ values for two sample t-test and ${ }^{\mathrm{b}}$ represents $\mathrm{P}$ values for $\chi 2$ test.

Figure 1 The workflow for spontaneous co-activation patterns (CAPs) analysis using the default mode network (DMN) as an example. A, A 6mm-sphere region of interest (ROI) centered on the peak seed locations (left cuneus (MNI coordinates: $0,-72,33$ )) for the DMN was specified. B, Time points (fMRI frames) in the time course of posterior cingulate cortex (PCC) in 
DMN were selected when their BOLD signals were greater than a preset threshold. The spatial correlation (Pearson's correlation coefficient) between the average of the selected time frames and DMN templates using all time frames increases quickly when including more frames by lowering the threshold. C, Data distribution of patients with MDD in the principal data set using the principal component analysis (PCA). The data was reshaped to a two-dimensional matrix. Then the two-dimensional matrix was descended dimensions using PCA. The first three eigenvectors were selected and displayed. D, the extracted fMRI frames were further classified into 5 groups using the spectral clustering method. Next, the DMN-CAP of each group was generated by averaging all subjects within groups. E, the workflow for extracting the overall dominant CAP maps using DMN-CAPs.

Figure 2 The results of spontaneous co-activation pattern analysis within DMN in both groups (The principal data set). A, the optimal threshold of DMN was $12.9 \%$. B, The DMN pattern was decomposed into 2 DMN-CAP maps in patients with MDD, while that of healthy controls was decomposed into 4 DMN-CAP maps. C, significantly attenuated occurrence rate of distinct CAP maps and number of transitions of CAP maps were detected in patients; $* * * P<$ 0.001 .

Figure 3 The results of spontaneous co-activation pattern analysis within SN in both groups

(The principal data set). A, the optimal threshold of SN was $7.6 \%$. B, The SN pattern was decomposed into 9 SN-CAP maps in patients with MDD, while that of healthy controls was 
decomposed into 2 SN-CAP maps. C, significantly enhanced occurrence rate of distinct CAP maps and number of transitions of CAP maps were detected in patients; *** $\mathrm{P}<0.001$.

Figure 4 The results of spontaneous co-activation pattern analysis within LCCN in both groups (The principal data set). A, the optimal threshold of LCCN was $11.2 \%$. B, The LCCN pattern was decomposed into 2 LCCN-CAP maps in patients with MDD, while that of healthy controls was decomposed into 2 LCCN-CAP maps. C, significantly attenuated within-cluster similarity were observed in patients; $* * * \mathrm{P}<0.001$.

Figure 5 The results of spontaneous co-activation pattern analysis within RCCN in both groups (The principal data set). A, the optimal threshold of RCCN was $10 \%$. B, The RCCN pattern was decomposed into 2 LCCN-CAP maps in patients with MDD, while that of healthy controls was decomposed into $3 \mathrm{RCCN}$-CAP maps. C, significantly reduced occurrence rate of distinct CAP maps was reported in patients; $* * * \mathrm{P}<0.001$.

Figure 6 the spatial expression of distinct overall dominant CAP maps of A, DMN, B, SN, C, LCCN and D, RCCN in both groups (The principal data set and the replication dataset). 


\section{Figures}

$\boldsymbol{A}$
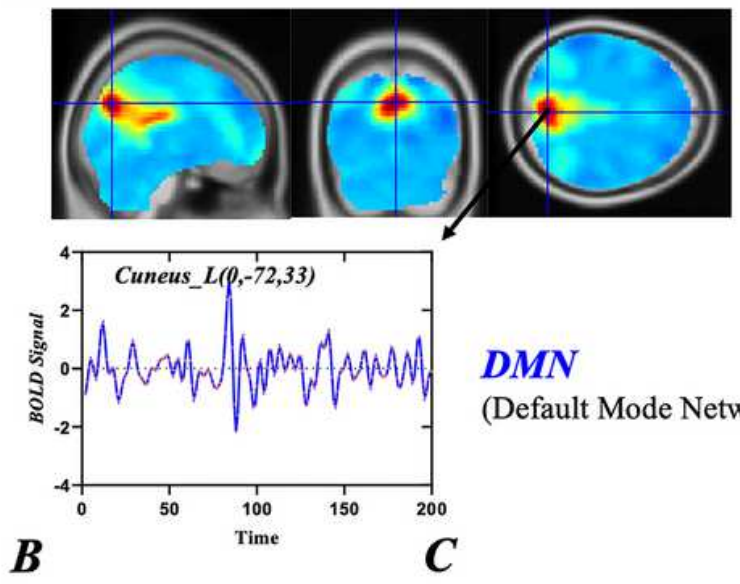

$D M N$

(Default Mode Network)

C
D
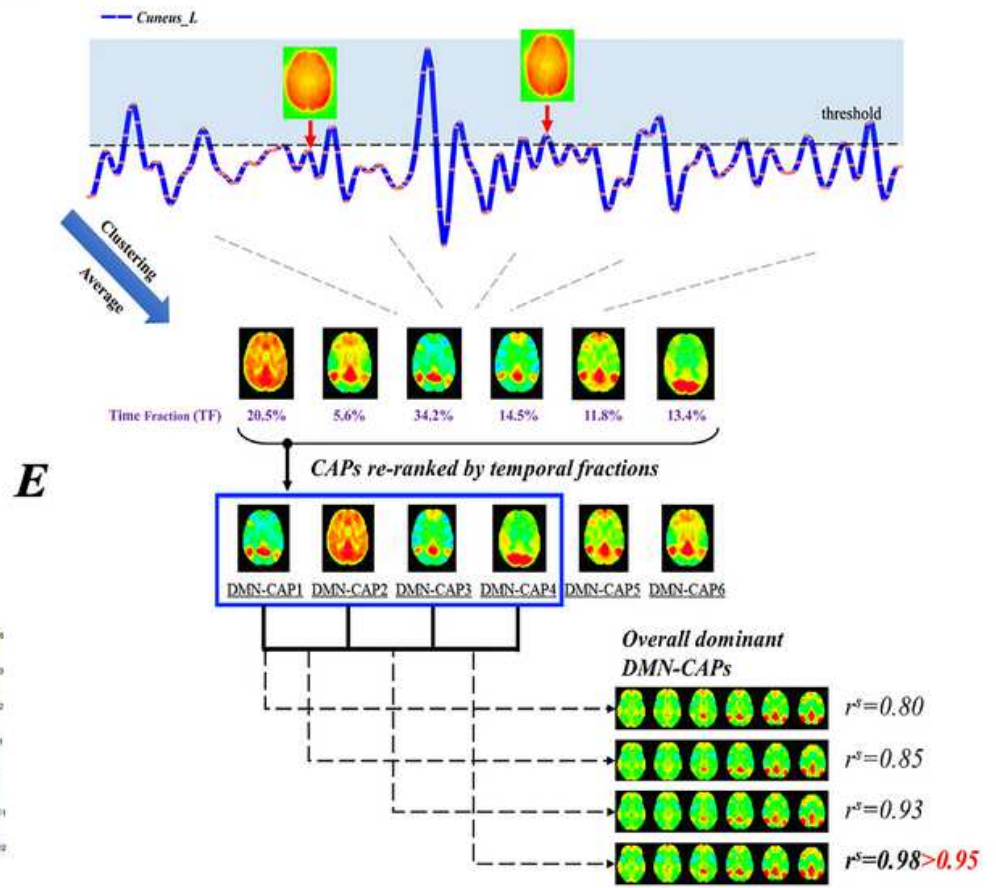

Figure 1

The workflow for spontaneous co-activation patterns (CAPs) analysis using the default mode network (DMN) as an example. A, A $6 \mathrm{~mm}$-sphere region of interest (ROI) centered on the peak seed locations (left cuneus (MNI coordinates: $0,-72,33)$ ) for the DMN was specified. B, Time points (fMRI frames) in the time course of posterior cingulate cortex (PCC) in DMN were selected when their BOLD signals were greater than a preset threshold. The spatial correlation (Pearson's correlation coefficient) between the average of the selected time frames and DMN templates using all time frames increases quickly when including more frames by lowering the threshold. C, Data distribution of patients with MDD in the principal data set using the principal component analysis (PCA). The data was reshaped to a two-dimensional matrix. Then the two-dimensional matrix was descended dimensions using PCA. The first three eigenvectors were selected and displayed. D, the extracted fMRI frames were further classified into 5 groups using the spectral clustering method. Next, the DMN-CAP of each group was generated by averaging all subjects within groups. E, the workflow for extracting the overall dominant CAP maps using DMN-CAPs. 
$\boldsymbol{A}$

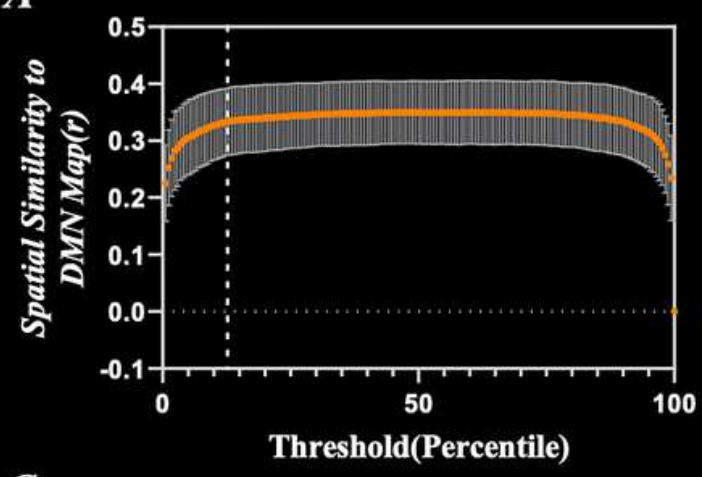

C

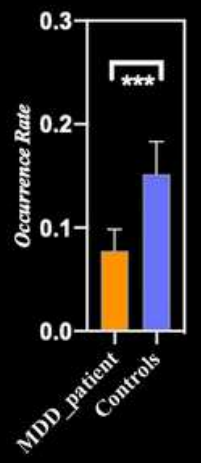

$\boldsymbol{B}$

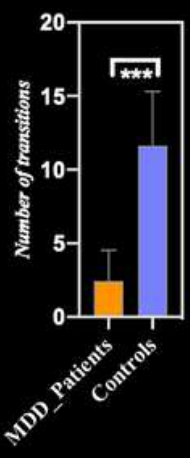

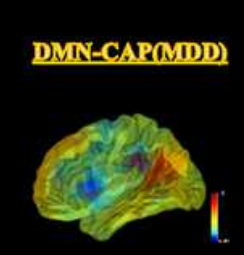

Overall average of $12.9 \%$ data
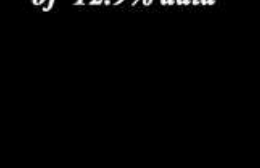

DMN-CAP(Controla)

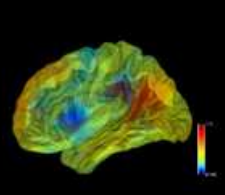

Overall average of $12.9 \%$ data

\section{Figure 2}

The results of spontaneous co-activation pattern analysis within DMN in both groups (The principal data set). A, the optimal threshold of DMN was $12.9 \%$. B, The DMN pattern was decomposed into 2 DMN-CAP maps in patients with MDD, while that of healthy controls was decomposed into 4 DMN-CAP maps. C, significantly attenuated occurrence rate of distinct CAP maps and number of transitions of CAP maps were detected in patients; $* \star * P<0.001$. 


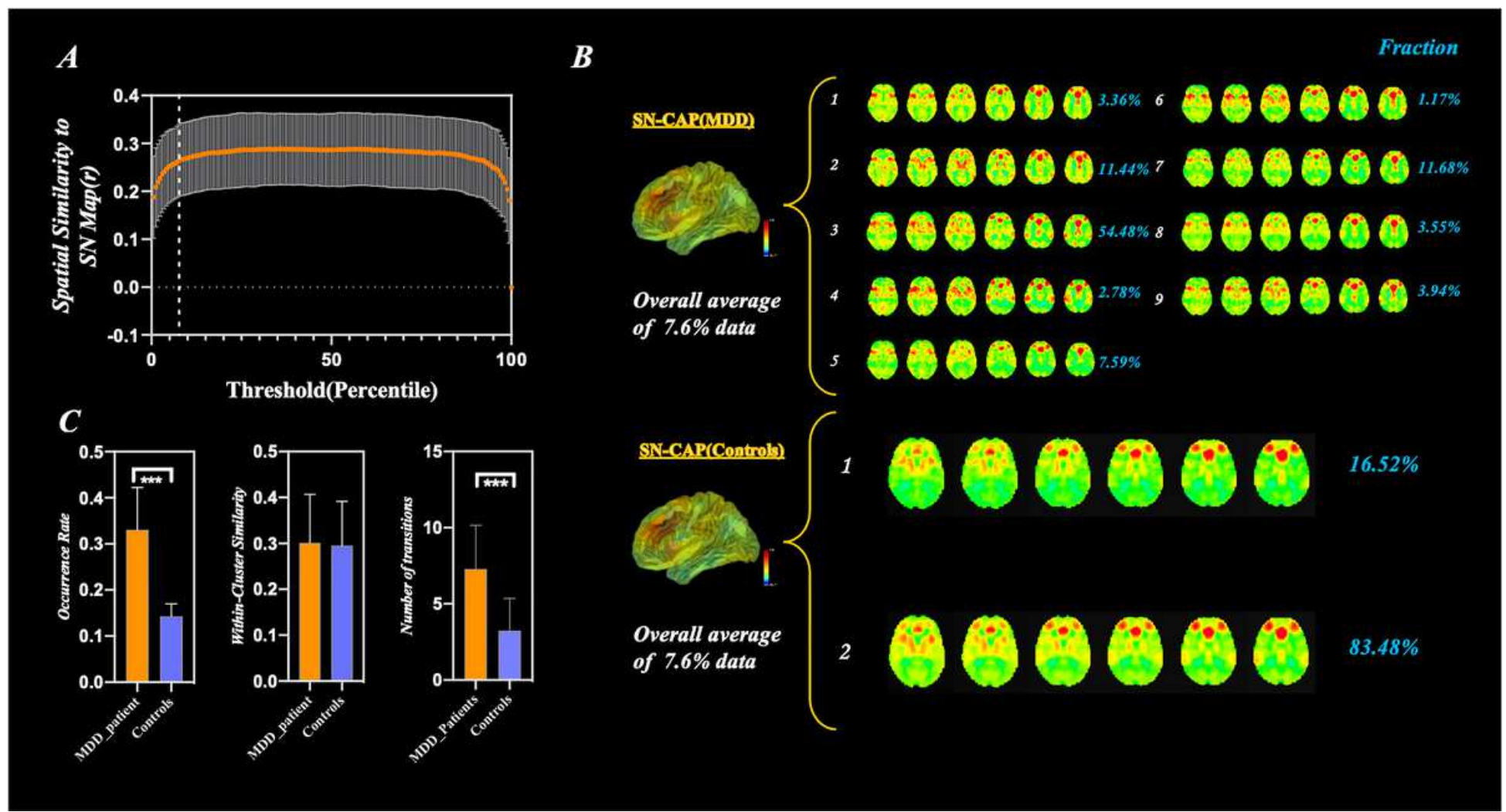

\section{Figure 3}

The results of spontaneous co-activation pattern analysis within $\mathrm{SN}$ in both groups (The principal data set). A, the optimal threshold of SN was $7.6 \%$. B, The SN pattern was decomposed into 9 SN-CAP maps in patients with MDD, while that of healthy controls was decomposed into 2 SN-CAP maps. C, significantly enhanced occurrence rate of distinct CAP maps and number of transitions of CAP maps were detected in patients; $* \star \star ~ P<0.001$. 
$\boldsymbol{A}$

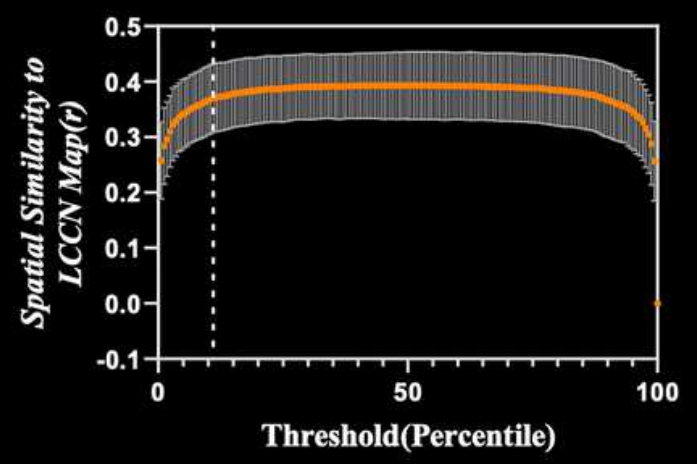

C
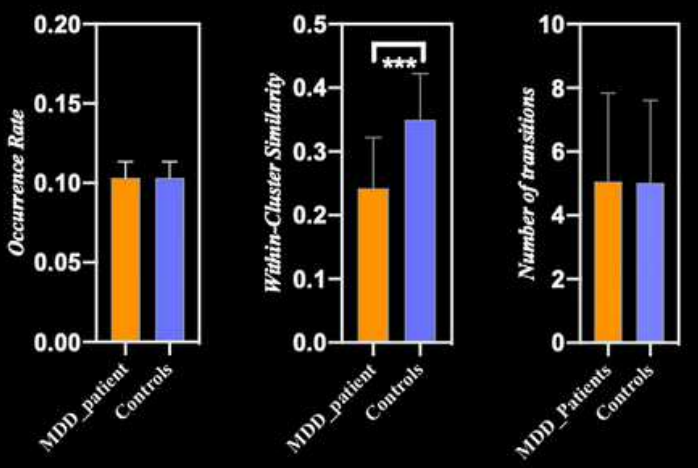

$\boldsymbol{B}$

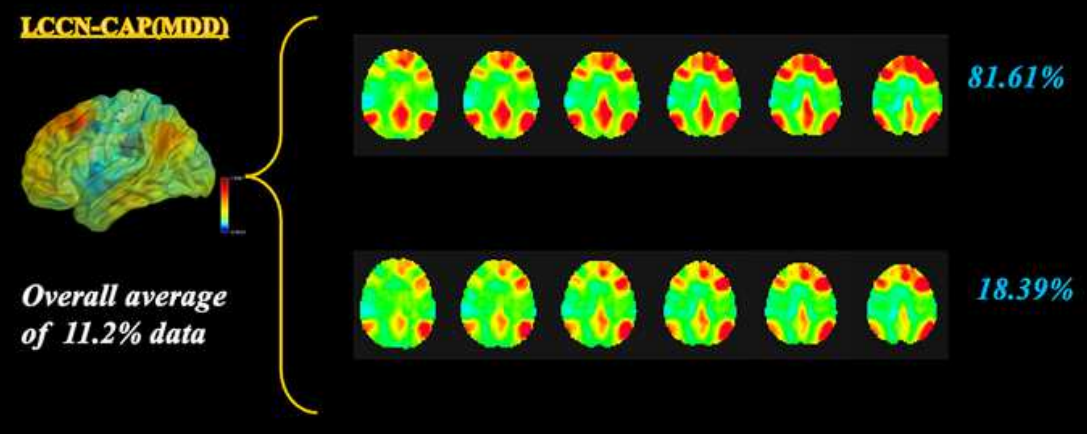

ICCN-CAP(Controls)

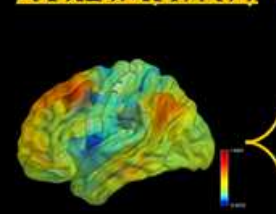

Overall average

of $11.2 \%$ data

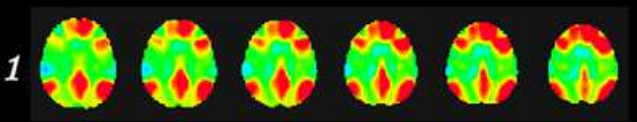

$81.79 \%$

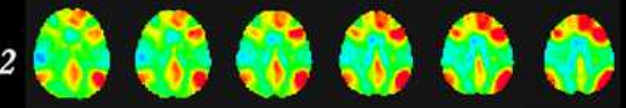

\section{Figure 4}

The results of spontaneous co-activation pattern analysis within LCCN in both groups (The principal data set). A, the optimal threshold of LCCN was $11.2 \%$. B, The LCCN pattern was decomposed into 2 LCCN-CAP maps in patients with MDD, while that of healthy controls was decomposed into 2 LCCN-CAP maps. C, significantly attenuated within-cluster similarity were observed in patients; $* \star \star ~ P<0.001$. 


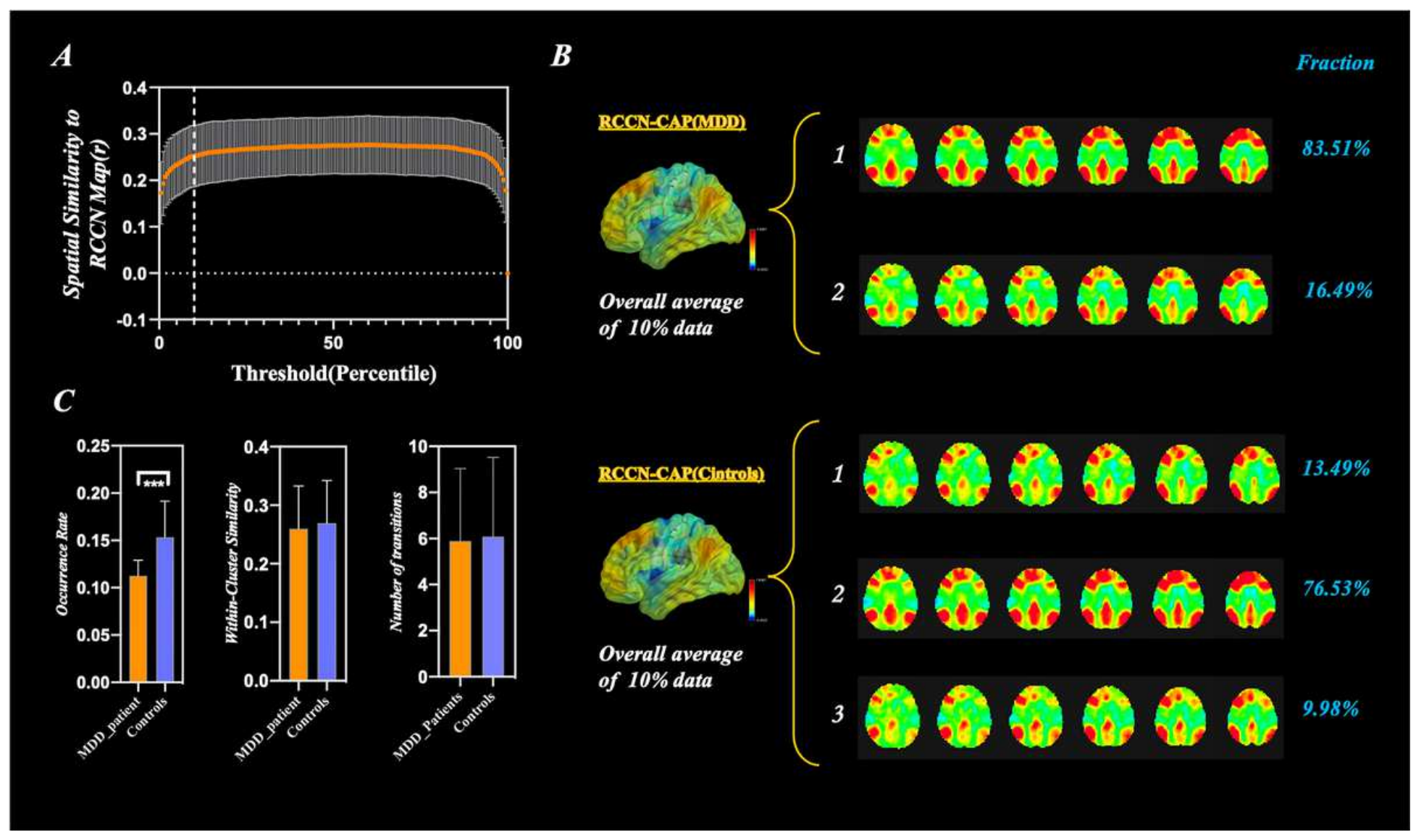

\section{Figure 5}

The results of spontaneous co-activation pattern analysis within RCCN in both groups (The principal data set). A, the optimal threshold of RCCN was $10 \%$. B, The RCCN pattern was decomposed into 2 LCCN-CAP maps in patients with MDD, while that of healthy controls was decomposed into 3 RCCN-CAP maps. C, significantly reduced occurrence rate of distinct CAP maps was reported in patients; ${ }^{* \star \star} P<0.001$. 
$\mathbf{A}$

Princpal Data Set

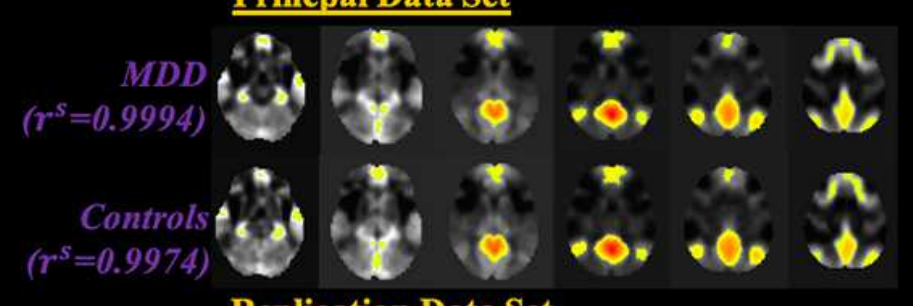

$\underline{\text { Replication Data Set }}$

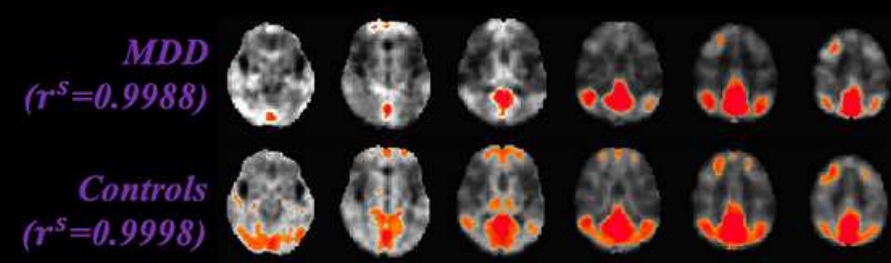

Overall dominant DMN-CAP-sets

C

\section{Princpal Data Set}
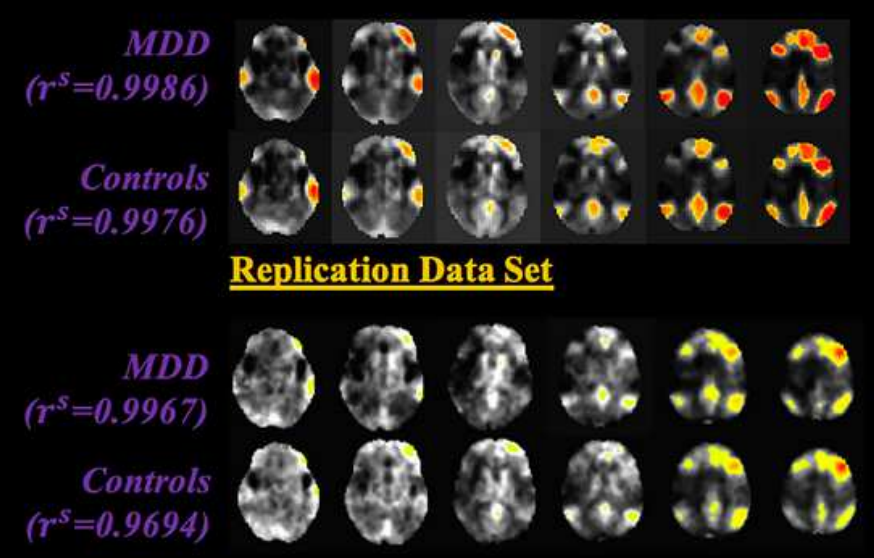

Overall dominant LCCN-CAP-sets
B

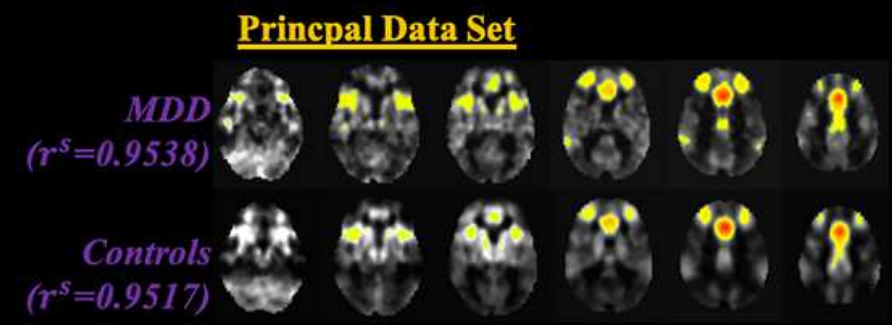

Replication Data Set

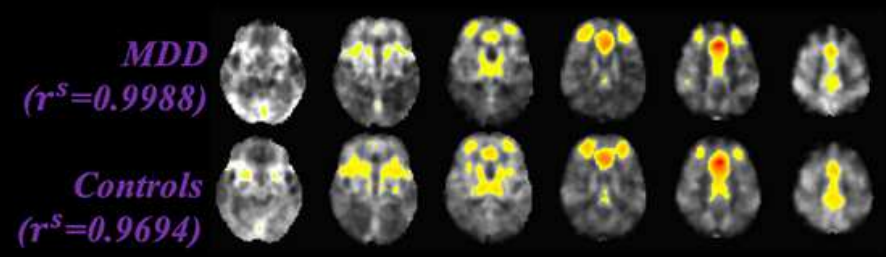

Overall dominant SN-CAP-sets

D

\section{Princpal Data Set}

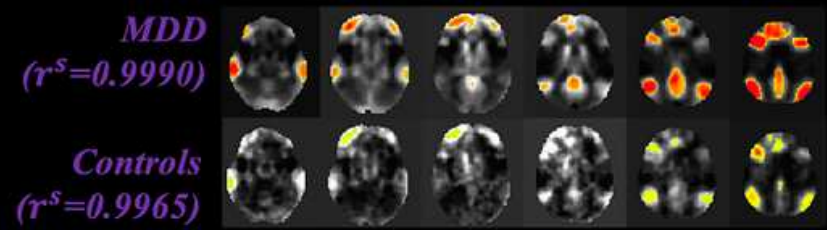

Replication Data Set

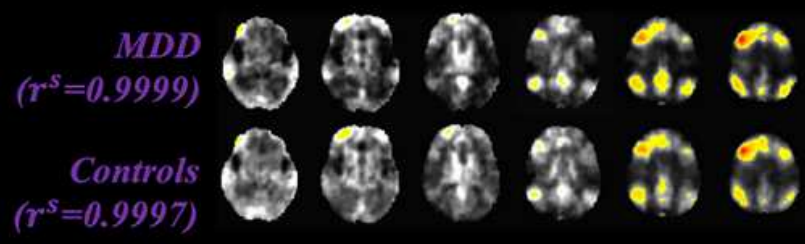

Overall dominant RCCN-CAP-sets

Figure 6

the spatial expression of distinct overall dominant CAP maps of A, DMN, B, SN, C, LCCN and D, RCCN in both groups (The principal data set and the replication dataset).

\section{Supplementary Files}

This is a list of supplementary files associated with this preprint. Click to download.

- supplementaryInformation.docx

- SupplementaryFigure1.png

- SupplementaryFigure2.png 
- SupplementaryFigure3.png

- SupplementaryFigure4.png

- SupplementaryFigure5.png 